\title{
Bienlargements on generalized topological spaces
}

\section{CARlos CARpintero, NAMEgALESH RAJESH and ENNIS Rosas}

\section{ABSTRACT.}

The aim of this paper is to introduce and study the concept of bienlargement on generalized topological spaces.

\section{REFERENCES}

[1] Carpintero, C., Rajesh, N. and Rosas, E., Separation axioms on enlargements of generalized toplogies, Revista Integración, 32 (2014), No. 1, 19-26

[2] Császár, Á., Generalized topology, generalized continuity, Acta Math. Hungar., 96 (2002), 351-357

[3] Császár, Á., Generalized open sets in generalized topology, Acta Math. Hungar., 106 (2005), 53-66

[4] Császár, Á., Enlargements and generalized topologies, Acta Math. Hungar., 120 (2008), 351-354

[5] Kanibir, A and Sagiroglu, S., A note on enlargements and generalized neighbourhood systems, Acta Math. Hungar., 136 (2012), $270-274$

[6] Kim, Y. K and Min, W. K., Further remarks on enlargements of generalised topologies, Acta Math. Hungar., 135 (2012), 184-191

[7] Kim, Y. K and Min, W. K., Remarks on enlargements of generalised topologies, Acta Math. Hungar., 130 (2011), 390-395

UNIVERSIDAD DE ORIENTE

DEPARTAMENTO DE MATEMÁticas

CUMANÁ, VENEZUELA

E-mail address: carpintero.carlosegmail.com

DEPARTMENT OF MATHEMATICS

Rajah Serfoji Govt. College

THANJAVUR-613005

TAMILNADU, INDIA

E-mail address: nrajesh_topology@yahoo.co.in

UNIVERSIDAD DE ORIENTE

DEPARTAMENTO DE MATEMÁTICAS

CUMANÁ, VENEZUELA

E-mail address: ennisrafaelegmail.com 